\title{
Prostate cancer and Hedgehog signalling pathway
}

\author{
L. M. Antón Aparicio a,b, R. García Campelo a, J. Cassinello Espinosa ${ }^{\mathrm{c}}, \mathrm{M}$. \\ Valladares Ayerbes ${ }^{\mathrm{a}}$, M. Reboredo López ${ }^{\mathrm{a}}$, S. Díaz Prado ${ }^{\mathrm{d}}$, and G. Aparicio \\ Gallego $^{\mathrm{e}}$
}

${ }^{a}$ Medical Oncology Service. C.H.U. Juan Canalejo. A Coruña, Spain

${ }^{b}$ Department of Medicine. University of La Coruña. A Coruña, Spain

${ }^{c}$ Medical Oncology Service. Guadalajara University Hospital. Guadalajara, Spain

${ }^{d}$ Investigation and Oncology Research Unit. C.H.U. Juan Canalejo. A Coruña, Spain

${ }^{e}$ Department of Biology. Faculty of Science. Lisbon University. Lisbon, Portugal

\begin{abstract}
The Hedgehog $(\mathrm{Hh})$ family of intercellular signalling proteins have come to be recognised as key mediators in many fundamental processes in embryonic development. Their activities are central to the growth, patterning and morphogenesis of many different regions within the bodies of vertebrates. In some contexts, Hh signals act as morphogens in the dose-dependent induction of distinct cell fates within a target field, in others as mitogens in the regulation of cell proliferation or as inducing factors controlling the form of a developing organ. These diverse functions of Hh proteins raise many intriguing questions about their mode of action. Various studies have now demonstrated the function of $\mathrm{Hh}$ signalling in the control of cell proliferation, especially for stem cells and stem-like progenitors. Abnormal activation of the Hh pathway has been demonstrated in a variety of human tumours. Hh pathway activity in these tumours is required for cancer cell proliferation and tumour growth. Recent studies have uncovered the role for Hh signalling in advanced prostate cancer and demonstrated that autocrine signalling by tumour cells is required for proliferation, viability and invasive behaviour. Thus, Hh signalling represents a novel pathway in prostate cancer that offers opportunities for prognostic biomarker development, drug targeting and therapeutic response monitoring.
\end{abstract}

Key words

Hh signalling; Prostate development; Tumorigenesis; Prostate cancer 


\section{Hh signalling pathway}

A much simplified description of the Hedgehog ( $\mathrm{Hh}$ ) pathway includes the Hh ligands, their receptor and a few of the molecules that transduce the signal (Fig. 1). In mammals there are three distinct $\mathrm{Hh}$ ligands [1] with different expression patterns: Sonic Hedgehog (Shh), Indian Hedgehog (Ihh) and Desert Hedgehog (Dhh). All three Hh ligands bind the transmembrane receptor Patched1 (Ptch1). In the absence of Hh ligand, Ptch1 inhibits a second transmembrane protein Smoothened (Smo). Hh binding to Ptch1 relieves the repression of Smo and allows Smo to further transduce the Hh signal. This Hh signalling results in the activation of the Gli family of transcription factors: Gli1, Gli2 and Gli3. Gli1 and Ptch1, as well as the gene encoding the Hedgehog interacting protein (Hip), are Hh response genes, and their expression has been used as evidence for active Hh signalling. Expression of Ptch1 or Hip starts a negative feedback loop that shuts down Hh signalling by sequestering Hh ligand. In the cytoplasm the Suppressor of Fused $[\mathrm{Su}(\mathrm{Fu})]$ protein also inhibits transduction of the Hh signal.

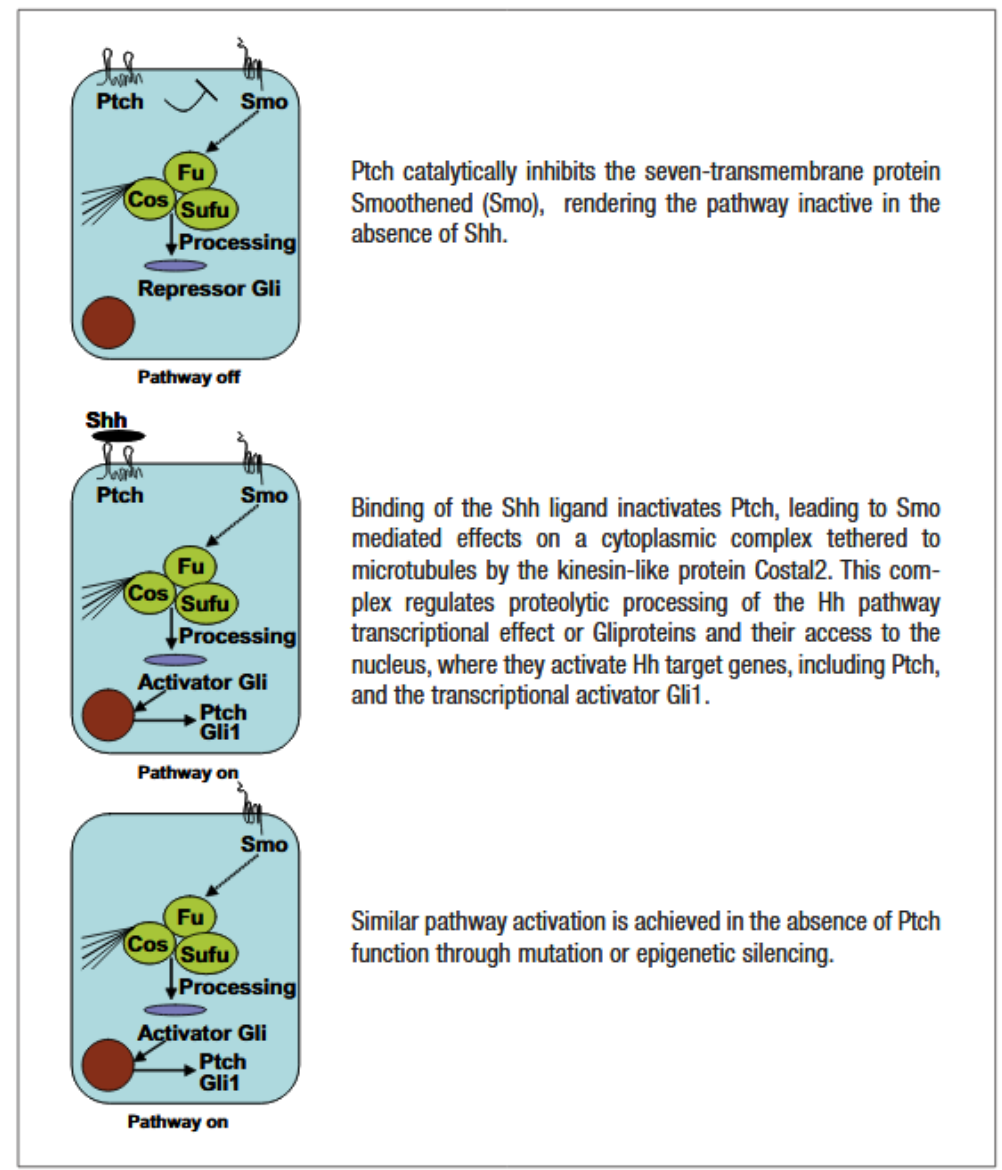

Fig. 1 A simplified model of Hh signalling. The Shh receptor, Patched (Ptch), is a 12transmembrane protein with homology to the resistance, nodulation, division (RND) bacterial transporter family. Costal2 (Cos), Kinase Fused (Fu), Suppressor of Fused ( $\mathrm{SuFu}$ (microtubule associated complex). The active Shh signalling peptide is formed by an autoprocessing reaction that converts a $45-\mathrm{kDa}$ protein into a $19-\mathrm{kDa}$ signalling peptide that is doubly lipid modified, with palmitate and cholesterol moieties at its $\mathrm{N}$ and $\mathrm{C}$ termini, respectively 
Although the precise cellular roles of Hh signals are still under scrutiny, it is clear that they can elicit different responses depending on the context in which the signals operate. Thus, Hh signals have been shown to regulate cell fate specification, cell proliferation and cell survival in different target cells. Signalling can be short- and long-range, direct and indirect (through the activation of a signalling relay), and importantly, concentration-dependent, evoking distinct molecular responses at discrete concentration thresholds (a classic morphogen activity).

The identification of many of the signalling factors that mediate cellular communication has led to two general conclusions. First, although there are many important signals, most of these fall into a few large families of secreted peptide factors: the Wingless-type (Wnt) [2], fibroblast growth factor [3], transforming growth factor (TGF)- $\beta$ superfamily [4], platelet-derived growth factor [5] and Hh families.

\section{Shh and prostate development}

The prostate gland is composed of a secretory epithelial parenchyma and a stroma consisting of smooth muscle cells and fibroblasts [6,7]. The stromal cells elaborate components of the extracellular matrix and paracrine factors that regulate epithelial cell proliferation and differentiation [8]. The role of mesenchyme in directing growth and morphogenesis during embryonic prostate development is well established [9] (Fig. 2).

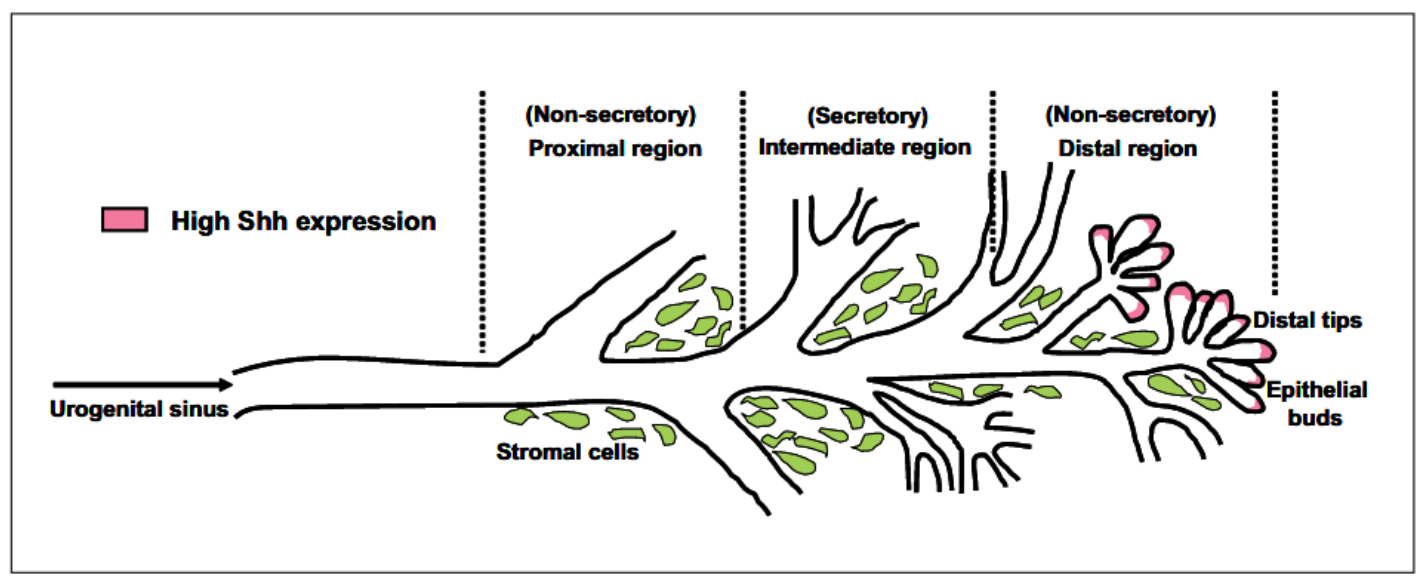

Fig. 2. The prostate originates from solid epithelial outgrowths that emerge from the endodermal urogenital sinus below the developing bladder. The prostatic buds grow into the adjacent mesenchyme, lengthen to form ducts, arborise and canalise. Considerable cellular heterogeneity exists across the prostatic ductal epithelium. Signals derived from the developing prostatic stroma are believed to control the rate and fate of proliferating prostate epithelial cells

The epithelial buds grow and form the prostate ducts. The prostate ducts will elongate and branch extensively both prenatally and postnatally until the final elaborate branching structure of the mature prostate is achieved. Hh function in the prostate seems to centre around the establishment of ductal branching. Shh expression begins in the epithelial layers of the rodent urogenital sinus and concentrates at areas of epithelial bud- ding [10]. Levels of Shh transcripts continue to be elevated as epithelial invaginations become ductal buds. During duct elongation Shh transcripts localise at the growing distal tips [10-12]. 
The role of Shh in regulating induction, proliferation and differentiation of the developing mammalian prostate has been studied most extensively in mouse and rat models.

During prenatal mouse prostate development, Shh activates Gli gene expression in the adjacent mesenchyme, and promotes epithelial proliferation and ductal growth [13-16]. Later in prostate development, Shh appears to inhibit growth and induce differentiation of epithelial cells into postmitotic, terminally differentiated luminal cells [17] (Fig. 3A). The dichotomous actions of Shh at different stages of prostate development may be due to differing concentrations of active Shh peptide or coregulators such as activin $\mathrm{A}$ and $\mathrm{TGFb} 1$ or a change in the state of stromal cell differentiation [17].

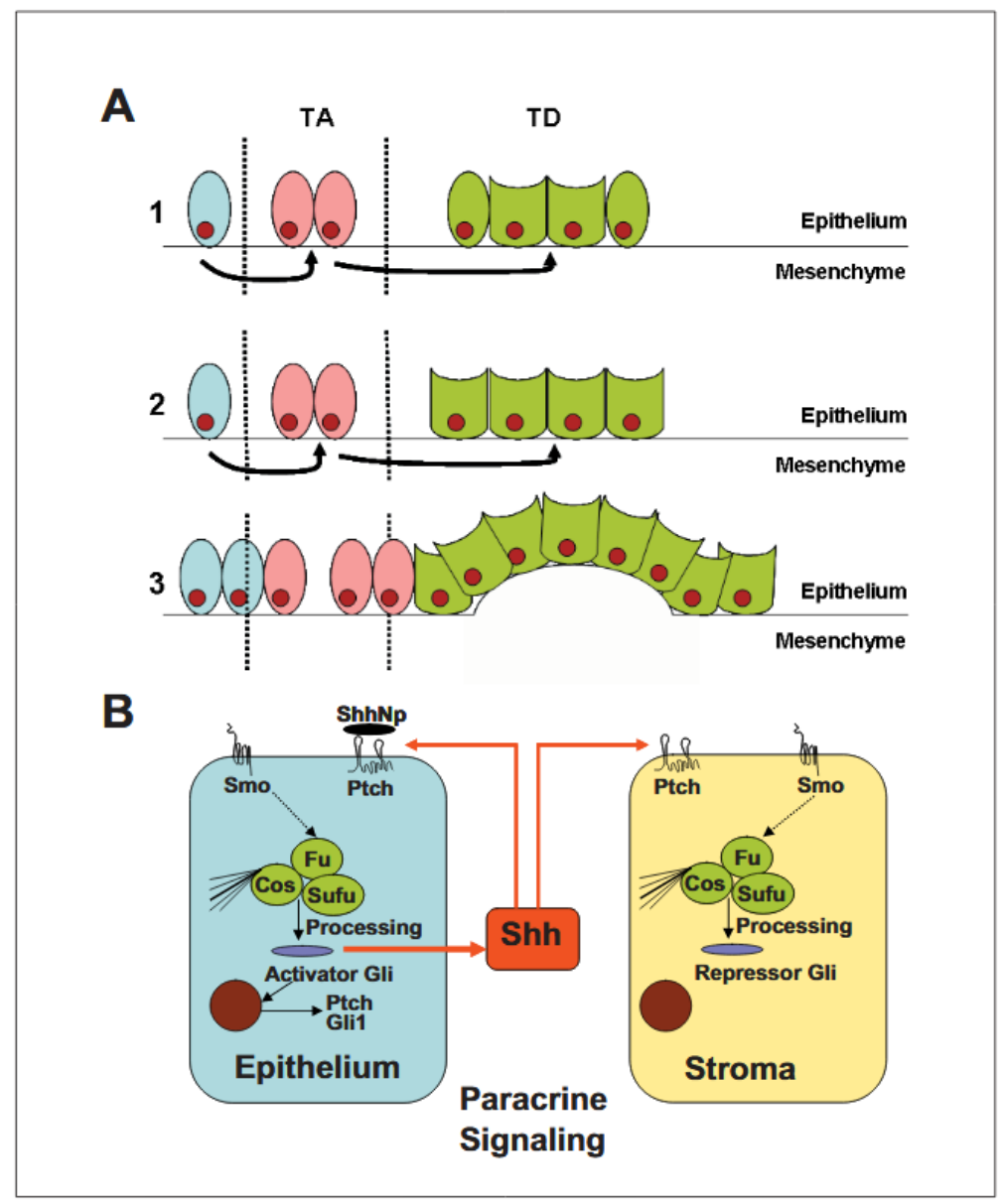

Fig. 3 A1 The initial formation of prostate buds does not require Shh signalling. 2 At prenatal prostate development, Shh promotes epithelial proliferation and ductal growth through Gli1 gen expression in the adjacent mesenchyme. 3 Later in prostate development, Shh appears to inhibit growth and induce differentiation of transit amplifying (TA) epithelial cells into postmitotic terminally differentiated (TD) luminal cells. B Epithelial-mesenchymal interactions driven by Shh. During normal prostate development Shh signalling is a paracrine event between the epithelium and the adjacent stroma 
The development of the prostate requires $\mathrm{Hh}$ signalling, although the initial formation of prostate buds does not require Shh. Shh is critical for maintaining appropriate prostate growth, proliferation and tissue polarity [18]. In the adult prostate, however, the activity of the Hh pathway is quite low [19]. Shh expression appears at approximately the same time as a surge in testosterone levels, suggesting that the two may be coordinated. Shh expression in the budding epithelium of the urogenital sinus correlates with a functional Hh pathway, as it results in upregulation of the Hh pathway proteins Ptch1, Gli1, Hip and bone morphogenetic protein (BMP)4 in the adjacent mesenchyme [10-12] (Fig. 3B).

Epithelial Shh expression is concentrated at sites of ductal bud formation and the tips of the growing ducts in which it activates Gli-1 and Gli-2 expression in the adjacent mesenchyme. Negation of Shh signalling impairs the activation of Gli-1 and Gli-2 expression and inhibits ductal outgrowth [13-15]. Because this effect entails primarily an inhibition of epithelial cell proliferation, it suggests that epithelial Shh expression activates stromal-mediated paracrine signals that feed back and stimulate epithelial cell proliferation [14] (Fig. 3B). Epithelialmesenchymal interactions driven by Hh ligand are a central theme in the morphogenesis of prostate growth (Table 1).

Table 1. Role of Shh between epithelial-mesenchymal interactions [25]

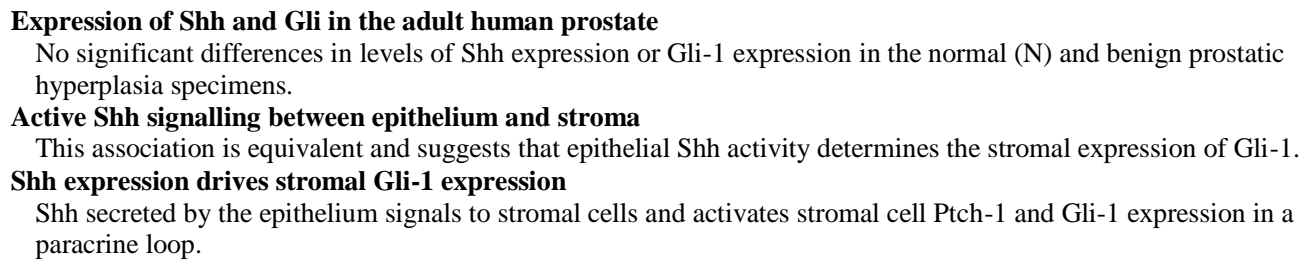

The role of the other mammalian Hh proteins is less clear: Dhh expression is not detected during the time of prostate development [13, 20], and low levels of Ihh were consistently observed.

\section{Shh signalling in prostate cancer}

As an important regulator of tissue polarity, active $\mathrm{Hh}$ signalling is required for ductal morphogenesis and proliferation during prostate development [21]. The adult prostate, on the other hand, does not contain active Hh signalling.

Recent studies showed a key role of Shh signalling in mediating endoderm-derived tissue formation, including prostate formation. Shh was expressed during prostatic branching morphogenesis, suggesting involvement in the initiation of androgen-dependent prostate development $[22,23]$, but analyses in mutant phenotypes revealed that Shh expression did not appear to be regulated by androgens. Androgen supplement was shown to reverse Shh mutant phenotypes [24], indicating that the role of Shh could be replaced by androgens during prostate development. 
In concordance with observations in other tumours, several lines of evidences have indicated Hh signalling in prostate tumorigenesis. A series of articles defined the role of Hh signalling in the growth and metastasis of advanced prostate cancer [25-28]. Molecular mechanisms and pathway components discovered in biochemical, genetic and molecular studies of Hh signalling during development, particularly in stem cell biology, are now demonstrated to be behind characteristics of tumour cells such as proliferation and invasive behaviour.

\section{In vitro models}

Prostate cancer cell lines, including PC3, LNCaP, DU145, 22RV1 and TSU, were shown to express high levels of $\mathrm{Hh}$ or its target genes [25-28]. Hh signalling modulated metastatic potential of rodent prostate cancer cell lines. Also when PrE cells, a putative prostate epithelium progenitor cell line, were stably transfected for Gli expression, the cells exhibited unlimited growth in vitro and formed aggressive subcutaneous tumours after transplantation into an athymic mouse [26].

\section{In vivo models}

A xenograft model was used to elucidate paracrine interactions between Shh-expressing human LNCaP tumour cells and host mouse stromal cells [25]. The LNCaP cells were genetically engineered to express Shh at high levels that dramatically accelerate tumour growth with increased Gli expression.

There are reported data showing Hh-induced in vivo prostate tumorigenesis in a mouse model induced by $\mathrm{Hh}$ overexpression that is initiated from normal status: Hh overexpression caused prostatic intraepithelial neoplasia (PIN) formation and the concomitant acceleration of prostate carcinoma metastatic activity. Overexpressions of $\mathrm{Hh}$ patterns were comparable to human conditions and gross morphological effects were similar [29].

These results indicated that the characteristics of PIN and prostate cancer formation were specifically due to Hh overexpression.

\section{Human profiles}

There are reports that activation of the Hh pathway occurs frequently in advanced human prostate cancer. High levels of Hh target genes, Ptch1 and Hip, are detected in over $70 \%$ of prostate tumours with Gleason scores $8-10$, but in only $22 \%$ of tumours with Gleason scores 3-6 [28].

Other researchers [30] observed abundant Gli-1 expression in $80 \%$ of prostate cancer tissues examined and speculated that Hh signalling could play an important role in prostate cancer. Shh pathway components, for example Gli, were detected in adult human prostate cancer, often with enhanced levels in tumours as compared to those in the normal status.

Activation of Hh signalling causes elevated expression of target genes Ptch1 and Hip. Thus, increased protein expression of Ptch1 and Hip indicates activation of the Hh expression, and thus the $\mathrm{Su}(\mathrm{Fu})$ inactivation appears to contribute to activation of Hh signalling in prostate tumours. As a negative regulator of the $\mathrm{Hh}$ pathway, $\mathrm{Su}(\mathrm{Fu})$ inhibits the function of Gli molecules, leading to inactivation of this pathway [31-33]. $\mathrm{Su}(\mathrm{Fu})$ is also reported to affect beta-catenin function [34]. 


\section{Shh and genetic relations}

Genetic mapping studies for familial prostate cancer have identified numerous chromosomal regions linked to prostate cancer susceptibility. On chromosome one a genetic association has been demonstrated between clinically significant prostate cancer and the brain tumour glioblastoma multiforme at 1p36 (Carcinoma Prostate Brain, CAPB), suggesting the presence of a common oncogene for these tumours [35-37].

It is interesting to note that multiple components of the Hh pathway are present within chromosomal regions associated with susceptibility to human prostate cancer (Fig. 4).

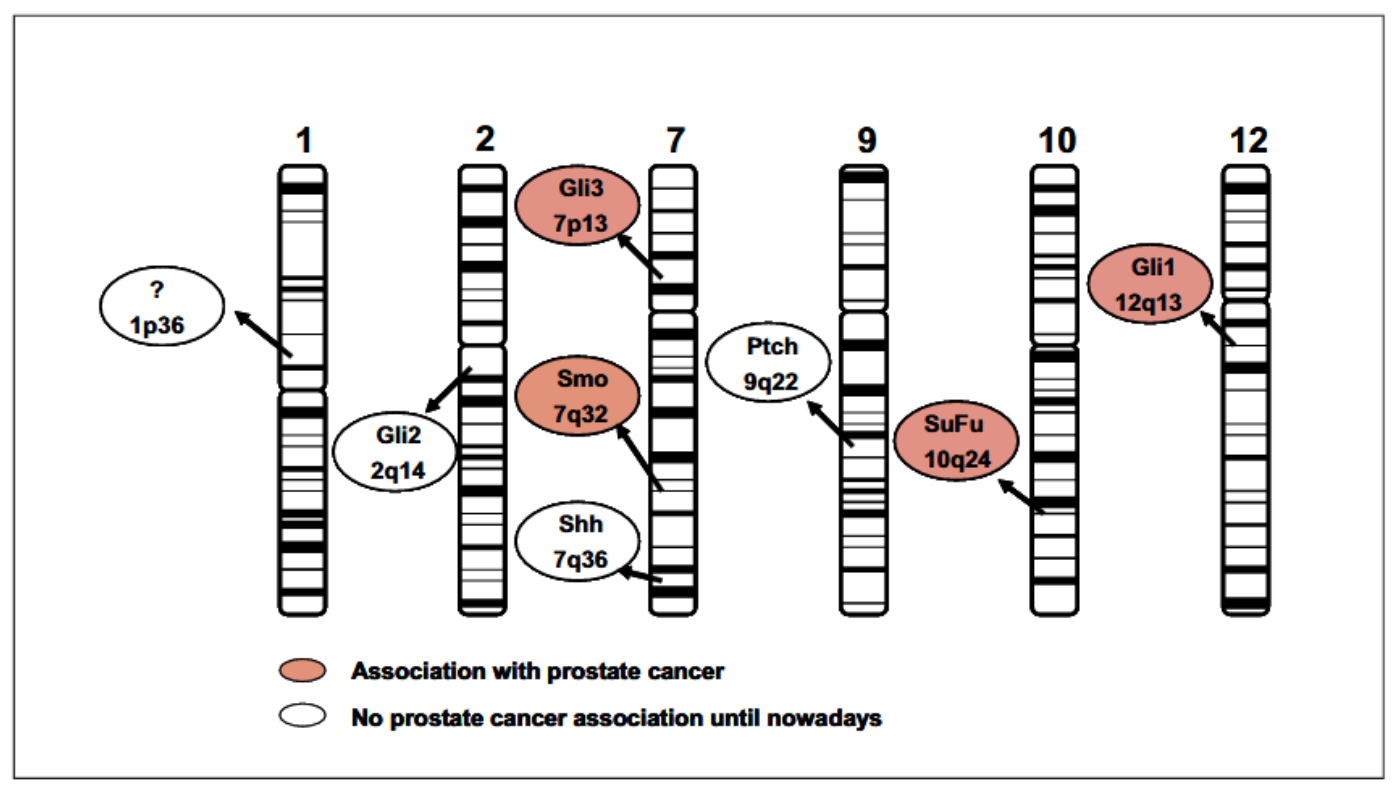

Fig. 4 Prostate cancer genetic associations and the Hh pathway. Adapted from Datta and Datta [59]

Analysis of data from genetic studies of familial prostate cancer showed that regions implicated in prostate cancer susceptibility contained the genes coding for Hh pathway components, including Gli1, Gli3, Smo and $\mathrm{Su}(\mathrm{Fu})$ [38-42].

In addition the $\mathrm{Su}(\mathrm{Fu})$ gene is localised at $10 \mathrm{q} 24$, a region with a frequent loss of heterozygosis $(\mathrm{LOH})$ in prostate cancer $[43,44]$.

Taken together, these findings suggest that activation of the Hh pathway involves prostate cancer progression. There might be several mechanisms by which the Hh pathway is activated in advanced prostate cancers, including loss of $\mathrm{Su}(\mathrm{Fu})$ protein expression, overexpression of Shh or other alterations (Table 2). 


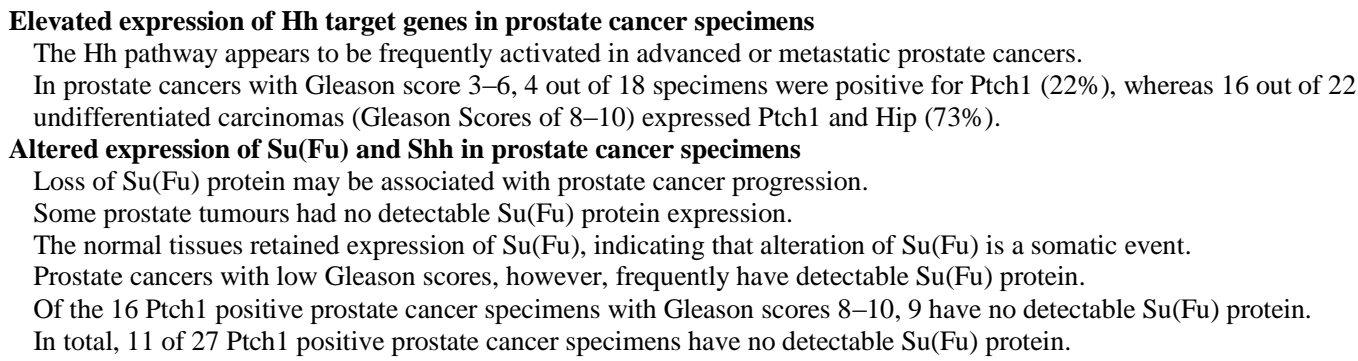

\section{Epithelial-mesenchymal interactions}

A growing body of evidence indicates that the stroma may undergo phenotypic and/or genotypic changes that enhance prostate cancer growth. Despite previous reports regarding involvement of $\mathrm{Hh}$ protein in the cancer formation within sites of epithelial-mesenchymal interactions, it has been reported that $\mathrm{Hh}$ can actually induce prostate cancer [45]. On the stromal side, evidence has been accumulated to indicate a "reactive stroma" that provides secretory factors that contribute to tumour growth and progression [46]. It has been shown that dedifferentiation of stromal smooth muscle [47] and distortion of epithelial cell lineage proportions [48] are factors in prostate carcinogenesis. Indeed, there are factors that are involved in both epithelial and stromal cells, with activities shifted between both sides or be- tween different forms of a same factor, in correlation with progression from normal to malignant stages [49-52]. In this study, it has been demonstrated that $\mathrm{Hh}$ overexpression caused prostate tumorigenesis from normal status to prostate cancer formation, and such transformation involved morphological characteristics within both the epithelial and the stromal sides. It has also been shown that Hh protein was detected within both the epithelial and the stromal cells during tumorigenesis, with concomitant loss of fibromuscular layer.

Experiments performed with the LNCaP xenograft tumour model demonstrated that Shh overexpression in LNCaP cells leads to increased tumour stromal Gli-1 expression and that Shh overexpression dramatically accelerates tumour growth. These data suggest that Hh signalling from prostate cancer cells to the stroma can elicit the expression of paracrine signalling, which pro- motes tumour growth [53]. However, a survey of adult human prostate tissues reveals substantial levels of Shh signalling in normal, hyperplasic and malignant prostate tissue. In cancer specimens, the Shh expression is localised to the tumour epithelium, whereas Gli-1 expression is localised to the tumour stroma. A tight correlation between the levels of Shh and Gli-1 expression suggests active signalling between the tissue layers.

Previous reports have shown that prostate cancer-associated stromal cells are phenotypically different from the stromal cells of the normal prostate, and the fact that reactive stroma can induce myofibroblast phenotype in prostate cancer $[8,54]$.

Studies of tumour-associated stromal cells have shown enhanced capacity to promote tumour growth in vivo $[55,56]$. More recently tumour-associated stromal cells and normal prostate stromal cells have been shown to exhibit different responses to paracrine stimulation with pigment epithelium-derived factor [57]. The mechanism for stromal activation has not been defined and the mechanisms by which stromal cells influence tumour growth are also not well understood. However, the distinctive character of the stromal compartment associated with prostate cancer may set the stage for a categorically different response to Shh signalling. Whereas Shh signalling in the stromal context of the normal prostate may help regulate the balance between proliferation and 
terminal differentiation, a similar level of signalling in the context of a cancer-associated reactive stroma may induce paracrine signals that promote tumour cell proliferation (Fig. 5).

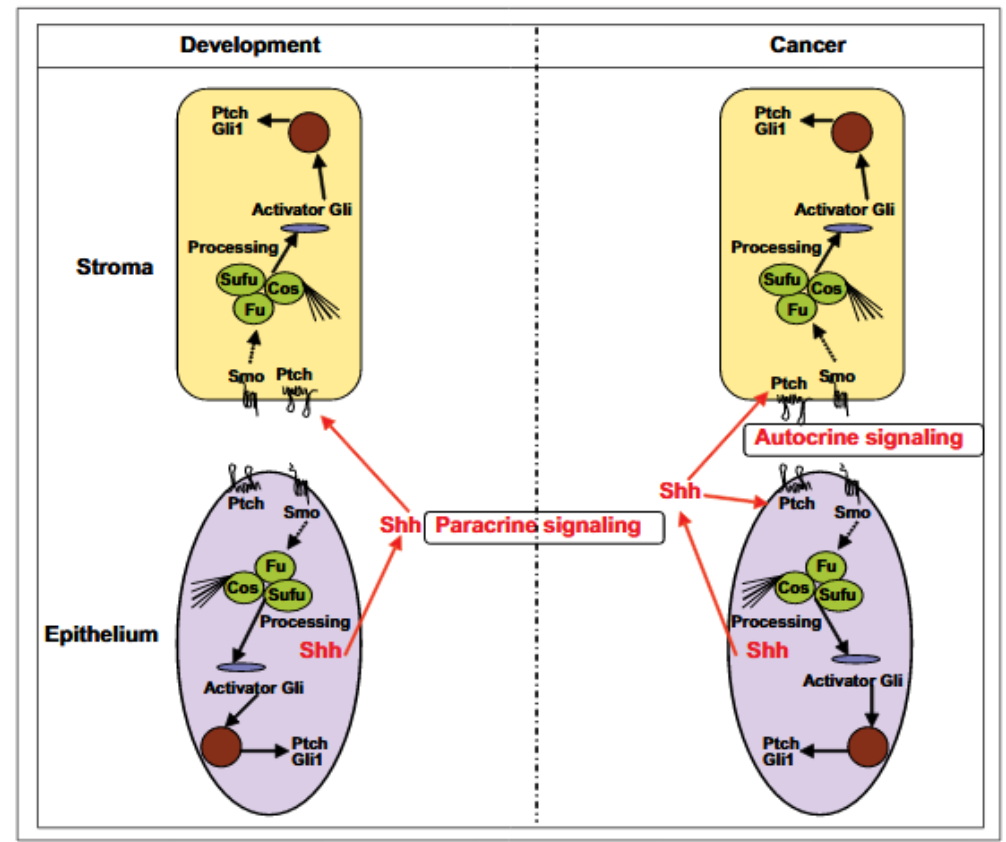

Fig. 5 Autocrine and paracrine signalling changes in $\mathrm{Hh}$ : during normal prostate development Shh signalling is a paracrine event between the epithelium and the adjacent stroma, while in prostate cancer tumours, Shh signalling appears to occur as an autocrine loop within cancerous epithelially derived cells. Adapted from Datta and Datta [59]

Tumours may be able to recruit normal stromal cells to support tumour growth by inducing expression of a variety of angiogenic and growth factors in a paracrine fashion [58]. The stromal reaction in cancer resembles the activity of stromal cells in wound healing and may create conditions that favour tumour progression.

\section{Conclusions}

The Hh signalling cascade is pivotal to embryonic development, and is involved in patterning a diverse range of vertebrate structures.

During foetal prostate development, Shh expression by the urogenital sinus epithelium activates Gli-1 expression in the adjacent mesenchyme and promotes out- growth of the nascent ducts. Shh signalling is downregulated at the conclusion of prostate ductal development.

Studies of prostate development suggest that $\mathrm{Hh}$ signalling is critical for normal ductal morphogenesis [15, 27, 28]. Epithelial Shh expression is concentrated at sites of ductal bud formation and the tips of the growing ducts in which it activates Gli-1 and Gli2 expression in the adjacent mesenchyme. Negation of Shh signalling impairs the activation of Gli-1 and Gli2 expression and inhibits ductal outgrowth. Because this effect entails primarily an inhibition of 
epithelial cell proliferation, it suggests that epithelial Shh expression activates stromal-mediated paracrine signals that feed back and stimulate epithelial cell proliferation [15].

Hh is a regulatory protein during embryonic development and its abnormal activation in adult tissues has been implicated in tumorigenesis within sites where epithelial-mesenchymal interactions take place. In the prostate, Hh signalling activation was observed during advanced cancer progression and metastasis, but whether $\mathrm{Hh}$ overexpression can initiate prostate tumorigenesis remains unknown.

Hh pathway genes in human prostate tissue reveal significant maintenance levels of Shh and Gli gene expression in the normal adult human prostate and comparably high levels of $\mathrm{Hh}$ signalling in prostate cancer and benign prostatic hyperplasia.

In cancer specimens, the Shh expression is localised to the tumour epithelium, whereas Gli-1 expression is localised to the tumour stroma. Tight correlation between the levels of Shh and Gli-1 expression suggests active signalling between the tissue layers. These data suggest that $\mathrm{Hh}$ signalling from prostate cancer cells to the stroma can elicit the expression of paracrine signals, which promote tumour growth.

Thus, while currently only speculative, the possibility exists that mutations or changes at multiple levels of the Hh pathway may lead to an increased risk of prostate cancer. This is coupled with data that indicate that the Hh pathway is present and expressed in human prostate tumours. Analysis of Shh protein immunoreactivity in both normal prostate tissues and tumours shows that it was demonstrated and upregulated with the more aggressive tumours, with the Shh-positive tumours having a higher Gleason grade.

The identification of signalling pathways that regulate the growth of advanced prostate cancer, in particular of androgen-independent tumour cells, is of utmost importance. These signalling pathways may represent both biomarkers for the development of advanced androgen refractory prostate cancer and targets for drug design that could be used, alone or together with hormonal treatments, to stop the growth of advanced prostate cancer.

\section{Acknowledgements}

We thank A. Carro-Ramos for typing the manuscript. S. Díaz Prado is supported by an Isidro Parga Pondal research contract by Xunta de Galicia (A Coruña, Galicia, Spain).

\section{References}

1. Echelard Y, Epstein DJ, St-Jacques B et al (1993) Sonic hedgehog, a member of a family of putative signalling molecules, is implicated in the regulation of CNS polarity Cell 75:1417-1430

2. Wodarz A, Nusse R (1998) Mechanisms of Wnt signalling in development. Annu Rev Biol 14:59-88

3. Szebenyi G, Fallon J (1999) Fibroblast growth factors as multifunctional signalling factors. Int Rev Cytol 185:45-106

4. Massague J, Chen Y (2000) Controlling TGF- $\beta$ signalling. Genes Dev 14:627-644

5. Betsholtz C, Karlsson L, Lindhl P (2001) Developmental roles of platelet-derived growth factors. Bioessays 23:494-507

6. McNeal JE (1988) Normal histology of the prostate. Am J Surg Pathol 12:619-633.

7. Castellucci E, Prayer-Galetti T, Roelofs M et al (1996) Cytoskeletal and cytocontractile protein composition of stromal tissue in normal, hyperplastic, and neoplastic human prostate. An immunocytochemical study with monoclonal antibodies. Ann NY Acad Sci 784:496-508

8. Rowley D (1998-1999) What might a stromal response mean to prostate cancer progression? Cancer Metastasis Rev 17:411-419

9. Cunha GR, Donjacour AA, Cooke PS et al (1987) The endocrinology and developmental biology of the prostate. Endocr Rev 8:338-362 
10. Lamm ML, Catbagan WS, Laciak RJ et al (2002) Sonic hedgehog activates mesenchymal Glil expression during prostate ductal bud formation. Dev Biol 249:349-366

11. Berman DM, Desai N, Wang X et al (2004) Roles for Hedgehog signalling in androgen production and prostate ductal morphogenesis. Dev Biol 267:387-398

12. Pu Y, Huang L, Prins GS (2004) Sonic hedgehog-patched Gli signalling in the developing rat prostate gland: lobe-specific suppression by neonatal estrogens reduces ductal growth and branching. Dev Biol 273:257-275

13. Podlasek CA, Barnett DH, Clemens JQ et al (1999) Prostate development requires Sonic hedgehog expressed by the urogenital sinus epithelium. Dev Biol 209:28-39

14. Lamm MLG, Catbagan WS, Laciak RJ et al (2002) Sonic hedgehog activates mesenchymal GLI1 expression during prostate ductal bud formation. Dev Biol 249:349-366

15. Berman DM, Desai N, Wang X et al (2004) Roles for Hedgehog signalling in androgen production and prostate ductal morphogenesis. Dev Biol 15:387-398

16. Horoszewicz JS, Leong SS, Kawinski E et al (1983) LNCaP model of human prostate carcinoma. Cancer Res 43:1809-1818

17. Wang B, Shou J, Ross S et al (2003) Inhibition of epithelial ductal branching in the prostate by Sonic hedgehog is indirectly mediated by stromal cells. J Biol Chem 278:18506-18513

18. Barnett DH, Huang HY, Wu XR et al (2002) The human prostate expresses sonic hedgehog during fetal development. J Urol 168:2206-2210

19. Freestone SH, Marker P, Grace OC et al (2003) Sonic hedgehog regulates prostatic growth and epithelial differentiation. Dev Biol 264:352-362

20. Bitgood MJ, McMahon AP (1995) Hedgehog and Bmp genes are coexpressed at many diverse sites of cell-cell interaction in the mouse embryo. Dev Biol 172:126-138

21. Freestone SH, Marker P, Grace OC et al (2003) Sonic hedgehog regulates prostatic growth and epithelial differentiation. Dev Biol 264:352-362

22. Podlasek CA, Barnett DH, Clemens JW et al (1999) Prostate development requires Sonic hedgehog expressed by the urogenital sinus epithelium. Dev Biol 209:28-39

23. Lamm ML, Catbagan WS, Laciak RJ et al (2002) Sonic hedgehog activates mesenchymal Glil expression during prostate ductal bud formation. Dev Biol 249:349-366

24. Berman DM, Desai N, Wang X et al (2004) Roles for Hedgehog signalling in androgen production and prostate ductal morphogenesis. Dev Biol 267:387-398

25. Fan L, Pepicelli CV, Dibble CC et al (2004) Hedgehog signalling promotes prostate xenograft tumor growth. Endocrinology 145:3961-3970

26. Karhadkar SS, Bova GS, Abdallah N et al (2004) Hedgehog signalling in prostate regeneration, neoplasia and metastasis. Nature 431:707-712

27. Sanchez P, Hernandez A, Stecca B et al (2004) Inhibition of prostate cancer proliferation by interference with Hedgehog-GLI1 signalling. Proc Natl Acad Sci USA 101:12561-12566

28. Sheng T, Li C, Zhang X et al (2004) Activation of the hedgehog pathway in advanced prostate cancer. Mol Cancer 3:29

29. Chen BY, Lin DP-C, Lin JY et al (2006) A mouse prostate cancer model induced by Hedgehog overexpression. J Biomed Sci 13:373-384

30. Dahmane N, Sanchez P, Gitton Y et al (2001) The Sonic Hedgehog-Gli pathway regulates dorsal brain growth and tumorigenesis. Development 128:5201-5212

31. Ding Q, Fukami S, Meng X et al (1999) Mouse suppressor of fused is a negative regulator of sonic hedgehog signalling and alters the subcellular distribution of Glil. Curr Biol 9:1119-1122

32. Kogerman P, Grimm T, Kogerman L et al (1999) Mammalian suppressor-of-fused modulates nuclearcytoplasmic shuttling of Gli-1. Nat Cell Biol 1:312-319

33. Stone DM, Murone M, Luoh S et al (1999) Characterization of the human suppressor of fused, a negative regulator of the zinc-finger transcription factor Gli. J Cell Sci 112:4437-4448

34. Meng X, Poon R, Zhang X et al (2001) Suppressor of fused negatively regulates beta-catenin signalling. J Biol Chem 276:40113-40119

35. Conlon EM, Goode EL, Gibbs M et al (2003) Oligogenic segregation analysis of hereditary prostate cancer pedigrees: evidence for multiple loci affecting age at onset. Int J Cancer 105:630-635

36. Gibbs M, Stanford JL, McIndoe RA et al (1999) Evidence for a rare prostate cancer-susceptibility locus at chromosome 1p36. Am J Hum Genet 64:776-787

37. Janer M, Friedrichsen DM, Stanford JL et al (2003) Genomic scan of 254 hereditary prostate cancer families. Prostate 57:309-319

38. Xu J, Dimitrov L, Chang BL et al (2005) A combined genomewide linkage scan of 1,233 families for prostate cancer-susceptibility genes conducted by the international consortium for prostate cancer genetics. Am J Hum Genet 77:219-229 
39. Dodge GR, Kovalszky I, Chu ML et al (1991) Heparan sulphate proteoglycan of human colon: partial molecular cloning, cellular expression and mapping of the gene (HSPG2) to the short arm of human chromosome 1. Genomics 10:673-680

40. Easton DF, Schaid DJ, Whittemore AS, Isaacs WJ (2003) Where are the prostate cancer genes? A summary of eight genome wide searches. Prostate 57:261-269

41. Kallunki P, Eddy RL, Byers MG et al (1991) Cloning of human heparin sulphate proteoglycan core protein, assignments of the gene (HSPG2) to 1p36.1-35 and identification of a BamHI restriction fragment length polymorphism. Genomics 57:389-396

42. Xu J, Gillanders EM, Isaacs SD et al (2003) Genome-wide scan for prostate cancer susceptibility genes in the Johns Hopkins hereditary prostate cancer families. Prostate 57:320-325

43. Latini JM, Rieger-Christ KM, Wang DS et al (2001) Loss of heterozygosity and microsatellite instability at chromosomal sites IQ and 10Q in morphologically distinct regions of late stage prostate lesions. J Urol 166:1931-1936

44. Leube B, Drechsler M, Muhlmann K et al (2002) Refined mapping of allele loss at chromosome 10q2326 in prostate cancer. Prostate 50:135-144

45. Chen BY, Lin DP, Liu JY et al (2006) A mouse prostate cancer model induced by Hedgehog overexpression. J Biomed Science 13:373-384

46. Sung SY, Chung LW (2002) Prostate tumorstroma interaction: molecular mechanisms and opportunities for therapeutic targeting. Differentiation 70:506-521

47. Wong YC, Tam NN (2002) Dedifferentiation of stromal smooth muscle as a factor in prostate carcinogenesis. Differentiation 70:633-645

48. Van Leenders GJ, Schalken JA (2003) Epithelial cell differentiation in the human prostate epithelium: implications of the pathogenesis and therapy of prostate cancer. Crit Rev Oncol Hematol 46:S3-S10

49. Fudge K, Bostwick DG, Stearns ME (1996) Platelet-derived growth factor A and B chains and the alpha and beta receptors in prostatic intraepithelial neoplasia. Prostate 29:282-286

50. Kaplan PJ, Mohan S, Cohen P et al (1999) The insulin-like growth factor axis and prostate cancer: lessons from the transgenic adenocarcinoma of mouse prostate (TRAMP) model. Cancer Res 59:2203-2209

51. Jin C, McKeeban K, Guo W et al (2003) Cooperation between ectopic FGFR1 and depression of FGFR2 in induction of prostatic intraepithelial neoplasia in the mouse prostate. Cancer Res 63:8784-8790

52. Evangelou AI, Winter SF, Huss WJ et al (2004) Steroid hormones, polypeptide growth factors, hormone refractory prostate cancer, and the neuroendocrine phenotype. J Cell Biochem 91:671-683

53. Fan L, Pepicelli CV, Dibble CC et al (2004) Hedgehog signalling promotes prostate xenograft tumor growth. Endocrinology 145:3961-3970

54. Tuxhorn JA, Ayala GE, Smith MJ et al (2002) Reactive stroma in human prostate cancer: induction of myofibroblast phenotype and extracellular matrix remodelling. Clin Cancer Res 8:2912-2923

55. Gunha GR, Hayward SW, Wang YZ (2002) Role of stroma in carcinogenesis of the prostate. Differentiation 70:473-485

56. Sung SY, Chung LW (2002) Prostate tumorstroma interaction: molecular mechanisms and opportunities for therapeutic targeting. Differentiation 70:506-521

57. Grayhack JT, Smith ND, Ilio K et al (2004) Pigment epithelium-derived factor, a human testis epididymis secretory product, promotes human prostate stromal cell growth in culture. J Urol 171:434-438

58. Hanahan D, Weinberg RA (2000) The hallmarks of cancer. Cell 100:57-70

59. Datta S, Datta MW (2006) Sonic Hedgehog signalling in advanced prostate cancer. Cell Mol Life Sci 63:435-448 